\title{
MicroRNA-128 targeting RPN2 inhibits cell proliferation and migration through the Akt-p53-cyclin pathway in colorectal cancer cells
}

\author{
TAICHENG ZHOU ${ }^{1 *}$, LILI WU ${ }^{2 *}$, QIRUI WANG ${ }^{3 *}$, ZHIPENG JIANG $^{1}$, YINGRU LI $^{1}$, NING MA $^{1}$, \\ WENHAO CHEN $^{1}$, ZEHUI HOU ${ }^{1}$, WENCHANG GAN ${ }^{1}$ and SHUANG CHEN ${ }^{1}$
}

\begin{abstract}
${ }^{1}$ Department of Gastroenterological Surgery and Hernia Center, Guangdong Provincial Key Laboratory of Colorectal and Pelvic Floor Diseases, The Sixth Affiliated Hospital of Sun Yat-sen University, Guangzhou, Guangdong 510655; ${ }^{2}$ Department of Ultrasonography, The Third Affiliated Hospital of Sun Yat-sen University, Guangzhou, Guangdong 510630;

${ }^{3}$ College of Traditional Chinese Medicine, Southern Medial University, Guangzhou, Guangdong 510515, P.R. China
\end{abstract}

Received October 17, 2017; Accepted April 17, 2018

DOI: $10.3892 / 01.2018 .9506$

\begin{abstract}
Colorectal cancer (CRC) is a malignancy with high metastatic rates. The mechanism of miR-128 on the regulation of Ribophorin-II (RPN2) in CRC cells was explored in the present study. Reverse transcription quantitative polymerase chain reaction (RT-qPCR) or western blot analyses were conducted to detect miR-128 and RPN2 levels in tissues and cell lines. AmiR-128 overexpression model was constructed using miR-128 mimic transfection in HT29 CRC cells. Then, cell proliferation was detected using a Cell Counting Kit- 8 assay, and the migratory and invasive abilities were measured by Transwell assay. RT-qPCR and western blot analysis were used to detect expression levels of protein kinase-B (Akt)-tumor protein 53 (p53)-cyclin pathway and metastasis-associated factors. In the present study, it was identified that aberrant decreased miR-128 was negatively correlated with RPN2 in CRC tissues. The increased RPN2 levels were significantly associated with poorly-differentiated histology, advanced stages and lymph nodes metastasis in patients with CRC. The survival rate of patients with CRC was also closely associated with RPN2 levels. In HT29 cells, miR-128 upregulation downregulated mRNA and protein levels of RPN2, and significantly inhibited cell proliferative, migratory and invasive abilities. Markedly decreased Akt
\end{abstract}

Correspondence to: Dr Shuang Chen, Department of Gastroenterological Surgery and Hernia Center, Guangdong Provincial Key Laboratory of Colorectal and Pelvic Floor Diseases, The Sixth Affiliated Hospital of Sun Yat-sen University, 26 Yuancun Erheng Road, Tianhe, Guangzhou, Guangdong 510655, P.R. China E-mail: shuangchen225@163.com

*Contributed equally

Key words: microRNA-128, ribophorin-II, colorectal cancer, cell proliferation, migration, invasion, protein kinase $\mathrm{B}$, matrix metalloproteinase phosphorylation and cyclin D1 levels and increased p53 levels were detected when cells were transfected with miR-128 mimics. Concurrently, decreased levels of matrix metalloproteinase (MMP)-2, MMP-9 and metastasis-associated protein 1 , and increased levels of epithelial-cadherin and tissue inhibitor of metalloproteinases 2 , were revealed in miR-128 mimic-transfected cells. Subsequent to screening with miRNA target prediction databases, the specificity of miR-128-targeted RPN2 was validated by a luciferase reporter assay. In conclusion, the results suggested that miR-128 was a specific negative regulator of RPN2, which regulated colorectal cancer cell proliferation and migration by affecting the Akt-p53-cyclin pathway. These data may provide novel evidence for the therapeutic potential of miR-128-based treatments for colorectal cancer.

\section{Introduction}

Colorectal cancer is one of the most serious malignancies threatening human health; it is ranked as the second leading cause of gastroenteric tumor-associated mortality worldwide, and the fourth leading cause of global tumor incidence and mortality (1). Due to increases in average population age, increasing CRC incidence has become a serious global public health issue $(2,3)$. As a result of a lack of effective diagnostic strategies for early-stage CRC, the majority of cases of CRC are diagnosed at advanced stages, and consequently are not able to be completely cured by surgical intervention. The 5-year survival rate of advanced-stage CRC is low, at only $10 \%(4,5)$. Tumor metastasis is one of the most common causes of cancer-associated mortality and involves cancer cells migrating from the primary tumor site to other organs via the lymphatic or circulatory systems. Although an increasing volume of evidence has demonstrated that the tumorigenesis rates and progression of CRC depends on various genetic variations (6-8), additional studies examining the in-depth molecular mechanisms are required. At present, novel molecular markers and targets for the early diagnosis of CRC are become an important area of study. 
MicroRNAs (miRNAs/miR), as a type of non-coding, single-stranded RNAs ranging from 21-24 nucleotides in length, have been previously demonstrated to be novel effective molecular targets for cancer therapy (9-12). The majority of miRNAs combine with the $3^{\prime}$ untranslated region (UTR) of target mRNAs through incomplete complementary combination, in the form of RNA-induced silencing complexes $(13,14)$. miRNAs inhibit the expression of target genes by suppressing mRNA translation or reducing the stability of mRNAs (15). An increasing number of studies have indicated that the aberrant expression of miRNAs may affect a number of physiological cell processes, including cell proliferation, differentiation, migration and invasion (16). In addition, miRNAs have also been demonstrated to be associated with clinical CRC development and metastasis by regulating the expression of target genes and relevant proteins (17).

Based on previous studies, miR-128 exists in a number of tissues with markedly different expression levels $(18,19)$. The aberrant expression of miR-128 is associated with numerous types of tumors, including glioma, prostate and breast cancer, and CRC (20). The overexpression of miR-128 may inhibit the developmental, proliferative and invasive abilities of prostate cancer cells (16). Concurrently, it may also decrease the proliferation rate of acute myeloid leukemia cells by increasing the extent of DNA damage (21). miR-128 was suggested to inhibit glioma cancer cell proliferation by suppressing the protein kinase B (Akt) pathway and cyclin-dependent kinase inhibitor 1 expression (22). Ai et al (17) identified that miR-128 inhibited murine CRC cell development in vitro and in vivo by targeting and inhibiting the expression of matrix metalloproteinase (MMP)3, MMP10 and MMP13 in CRC cells. Although the mechanisms of miR-128 in tumors have been studied extensively, its role in human CRC remains unclear.

Ribophorin-II (RPN2), encoding the essential subunit of the oligosaccharide transferase complex, is important for cell structure, cell signal recognition and transduction (23). The expression of RPN2 was identified to be increased in patients with gastric cancer, CRC, hepatocellular carcinoma, lung and breast cancer, and head and neck neoplasms, with a potential association with clinical phenotype (24). It is also associated with tumor metastasis, prognosis and drug resistance in tumor cells (25). RPN2 knockdown was demonstrated to promote cell apoptosis, inhibit cell proliferation and increase cell sensitivity to chemotherapeutics in lung and breast cancer, and osteosarcoma (26). RPN2 overexpression was also verified to inactivate glycogen synthase kinase $3 \beta$ and lead to a steady expression of mutational tumor protein (p53), and to regulate tumorigenesis and metastasis in breast cancer stem cells (27). Whether there is any association between miR-128 and RPN2 in regulating CRC is currently unknown, and merits additional study.

Collectively, the present study focused on the role of miR-128 in regulating RPN2 expression in CRC cells, and provides novel evidence for the therapeutic potential of miRNA regulating colorectal cancer.

\section{Materials and methods}

Patients and tumor samples. A total of 53 patients with CRC in different Tumor-Node-Metastasis stages, as proposed by
American Joint Committee on Cancer (28-31), aged between 32-74 years old (median, 57 years old), and admitted to The Sixth Affiliated Hospital of Sun Yat-sen University (Guangzhou, China) were enrolled from November 2015 to November 2016. No patient had received radiotherapy or chemotherapy prior to surgery. Matched adjacent normal colorectal tissues (taken $>2 \mathrm{~cm}$ away from the cancer tissue) were collected as negative controls. Basic clinical and pathological data were collected, and all patients provided informed consent. All tissue samples from patients were collected and protocols were performed according to the procedures approved by the Institutional Review Board of the Independent Ethics Committee of The Sixth Affiliated Hospital of Sun Yat-sen University (approval no. BZ20153587).

Cell culture and grouping. Human CRCHT29, SW480, SW620 and HCT116 cell lines and the normal human colon mucosa epithelia NCM460 cell line were all obtained from American Type Culture Collection (Manassas, VA, USA). Cells were cultured in RPMI-1640 medium (Hyclone; GE Healthcare Life Sciences, Logan, UT, USA) containing 10\% fetal bovine serum (FBS; Gibco; Thermo Fisher Scientific, Inc., Waltham, MA, USA) with $1 \%$ penicillin/streptomycin (Invitrogen; Thermo Fisher Scientific, Inc.) at $37^{\circ} \mathrm{C}$ in a $5 \% \mathrm{CO}_{2}$ incubator. The medium was changed once every 2 days. Cell passage was performed when cells reached $80 \%$ confluence. Cells in the logarithmic phase were used.

miR-128 mimic transfection. Human CRC HT29 cells were seeded onto 12 -well plates at a concentration of $5 \times 10^{4}$ cells/well and cultured for $24 \mathrm{~h}$ prior to transfection. Then, confluent cells were transfected with miR-128 mimics (5'-UUUCUCUGGCCAAGUGACACU-3') and control scrambled sequences (5'-GTGACCCACGAT GTGTATTCGC-3') (Shanghai GenePharma, Co., Ltd., Shanghai, China), at a final concentration of $50 \mathrm{nmol} / \mathrm{l}$ with Lipofectamine ${ }^{\circledR} 2000$ (Invitrogen; Thermo Fisher Scientific, Inc.) according to manufacturer's protocol.

Human CRC HT29 cells were randomly allocated into three groups: Control; mock (cells transfected with control scrambled sequences); and miR-128 (cells transfected with miR-128 mimics) groups. Reverse transcription quantitative polymerase chain reaction (RT-qPCR) was conducted to determine miR-128 expression levels, and RT-qPCR and western blot analyses were conducted to determine RPN2 expression levels in the control, mock and miR-128 groups, as described subsequently.

Cell Counting Kit (CCK)-8 assay. Cell viabilities in the control, mock andmiR-128 groups were measured using a CCK-8 assay (Beyotime Institute of Biotechnology, Haimen, China). Cells were seeded onto 96 -well plates $(100 \mu \mathrm{l} /$ well $)$ at $5 \times 10^{3}$ cells/well and cultured in RPMI-1640 medium for $4 \mathrm{~h}$ at $37^{\circ} \mathrm{C}$ in a $5 \% \mathrm{CO}_{2}$ incubator. Subsequently, $20 \mu \mathrm{l}$ CCK-8 reagent was added and cells were incubated at $37^{\circ} \mathrm{C}$ again for $1 \mathrm{~h}$. Optical density values were measured at a wavelength of $450 \mathrm{~nm}$ by the iMark microplate absorbance reader (Bio-Rad Laboratories, Inc., Hercules, CA, USA). 
Table I. Primer sequences used in the present study.

\begin{tabular}{|c|c|c|}
\hline Name & Direction & Sequences $\left(5^{\prime}-3^{\prime}\right)$ \\
\hline hsa-miR-128 & Forward & UCACAGUGAACCGGUCUCUUU \\
\hline \multirow[t]{2}{*}{ U6 } & Forward & TGCGGGTGCTCGCTTCGCAGC \\
\hline & Reverse & TGCGGGTGCTCGCTTCGCAGC \\
\hline \multirow[t]{2}{*}{ RPN2 } & Forward & AGGAAGTGGTGTTTGTTGCC \\
\hline & Reverse & ACAGTCGAGGGAGCTTCTTC \\
\hline \multirow[t]{2}{*}{ p53 } & Forward & TCAGTCTACCTCCCGCCATA \\
\hline & Reverse & TTACATCTCCСАAАCATCССТ \\
\hline \multirow[t]{2}{*}{ CyclinD1 } & Forward & CAATGACCCCGCACGATTTC \\
\hline & Reverse & AAGTTGTTGGGGCTCCTCAG \\
\hline \multirow[t]{2}{*}{ MMP-2 } & Forward & TGTGTTGTCCAGAGGCAATG \\
\hline & Reverse & ATCACTAGGCCAGCTGGTTG \\
\hline \multirow[t]{2}{*}{ MMP-9 } & Forward & TTTGAGTCCGGTGGACGATG \\
\hline & Reverse & GCTCCTCAAAGACCGAGTCC \\
\hline \multirow[t]{2}{*}{ MTA1 } & Forward & AAACTGCCCTGAGTGTGGT \\
\hline & Reverse & AAATATGTTGACCCAGCTCATCT \\
\hline \multirow[t]{2}{*}{ E-cadherin } & Forward & CTGAAGTGACTCGTAACGAC \\
\hline & Reverse & CATGTCAGCCAGCTTCTTGAAG \\
\hline \multirow[t]{2}{*}{ TIMP2 } & Forward & GCCTGACGGTCATATGGTAGA \\
\hline & Reverse & GAATGCGCCAAAAACCCCAT \\
\hline \multirow[t]{2}{*}{ GAPDH } & Forward & GAATGGGCAGCCGTTAGGAA \\
\hline & Reverse & AAAAGCATCACCCGGAGGAG \\
\hline
\end{tabular}

hsa, Homo sapiens; miR, microRNA; RPN2, Ribophorin-II; p53, tumor protein 53; MMP, matrix metalloproteinase; MTA1, metastasisassociated protein 1; E-cadherin, epithelial cadherin; TIMP1, Tissue inhibitor of metalloproteinases 2.

Transwell assay. HT29 cells $\left(6 \times 10^{4}\right.$ cells/well) were seeded in the upper wells of a Transwell migration system attached with polycarbonate filters (Corning Incorporated, Corning, NY, USA) in RPMI-1640 supplemented with $0.1 \%$ FBS. The lower wells were filled with RPMI-1640 with $10 \%$ FBS. Following incubation for $24 \mathrm{~h}$ at $37^{\circ} \mathrm{C}$, the non-migrating cells from the upper well were removed with cotton swabs. The cells that had migrated through the membranes were fixed with $70 \%$ cold ethanol at $4^{\circ} \mathrm{C}$ for $30 \mathrm{~min}$, and stained by $0.1 \%$ crystal violet at $37^{\circ} \mathrm{C}$ for $30 \mathrm{~min}$. Cells were counted in 5 separate fields of view and images were captured using light microscopy with x200 magnification (Olympus Corporation, Tokyo, Japan) and cell migration rate was calculated.

The cell invasion assay was performed as aforementioned, with the exception of the application of chambers with Matrigel $^{\circledR}$ (BD Biosciences, Franklin Lakes, NJ, USA), which was melted at $37^{\circ} \mathrm{C}$ for $30 \mathrm{~min}$ prior to usage.

Reverse transcription quantitative polymerase chain reaction $(R T-q P C R)$. Quantification of miR-128 was performed using a TaqMan MicroRNA Reverse Transcription kit (Thermo Fisher Scientific, Inc.). The expression of miR-128 was normalized using U6. Analysis of relative gene expression data was conducted using $2^{-\triangle \Delta C q}$ method (32).

To determine mRNA levels of RPN2, p53, Cyclin D1, MMP-2, MMP-9, epithelial-cadherin (E-cadherin), metastasis-associated protein 1 (MTA1) and tissue inhibitor of metalloproteinases 2 (TIMP2), total RNA extracted from
CRC tissues, different groups of CRC cells, or cells transfected with miR-128 mimics was firstly reverse transcribed using the Takara PrimeScript RT reagent kit (Takara Bio, Inc., Otsu, Japan) at $25^{\circ} \mathrm{C}$ for $5 \mathrm{~min}, 42^{\circ} \mathrm{C}$ for $60 \mathrm{~min}$ and $72^{\circ} \mathrm{C}$ for $10 \mathrm{~min}$. Quantification of mRNA was determined using a TaqMan Gene Expression Assay(Thermo Fisher Scientific, Inc.). The thermocycling conditions of the reactions were as follows: $15 \mathrm{sec}$ at $95^{\circ} \mathrm{C}$, followed by 40 cycles of denaturation at $95^{\circ} \mathrm{C}$ for $15 \mathrm{sec}$ and annealing/extension at $60^{\circ} \mathrm{C}$ for $25 \mathrm{sec}$. Target gene expression was normalized to GAPDH. The primer sequences are summarized in Table I.

Western blot analysis. Total protein of cells in each group was extracted using ProteoPrep ${ }^{\circledR}$ Total Extraction Sample kit (Sigma-Aldrich; Merck KGaA, Darmstadt, Germany). GAPDH was used as a control. Concentrations of proteins were determined using the Bradford assay (Bio-Rad Laboratories, Inc.), and protein samples (20 $\mu \mathrm{g} /$ lane) were separated on $10-15 \%$ SDS-PAGE and electroblotted onto polyvinylidene fluoride membranes (EMD Millipore, Billerica, MA, USA). Following blocking with $5 \%$ non-fat dry milk dissolved in $0.01 \mathrm{~mol} / 1$ TBST solution (Wuhan Boster Biological Technology, Ltd., Wuhan, China), for $1 \mathrm{~h}$ at $37^{\circ} \mathrm{C}$, membranes were incubated with specific primary antibodies overnight at $4^{\circ} \mathrm{C}$. Then, the membranes were treated with a goat anti-rabbit immunoglobulin G H\&L horseradish peroxidase-conjugated secondary antibody (Abcam; cat. no., ab6721, 1:5,000 dilution) at $37^{\circ} \mathrm{C}$ for $1 \mathrm{~h}$ and exposed to X-ray film. Finally, immunoreactive 
A

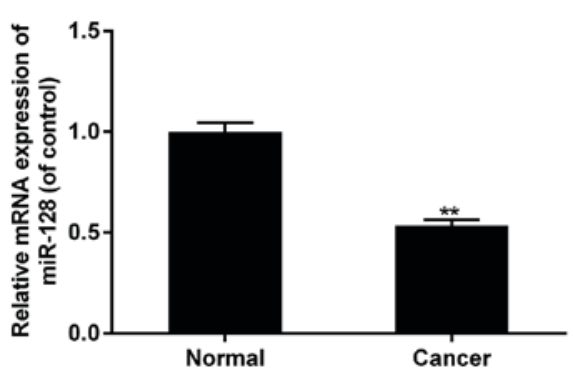

C

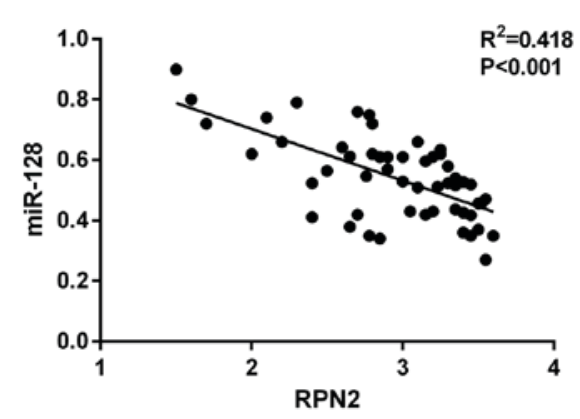

B

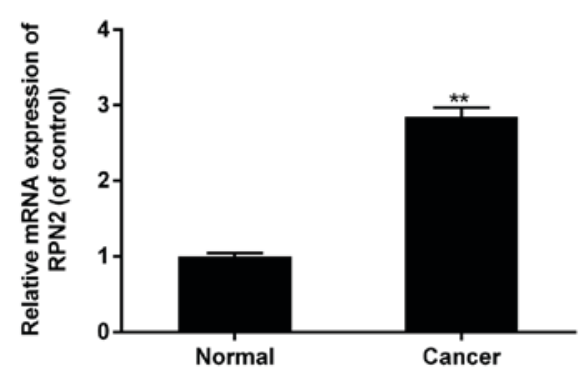

D

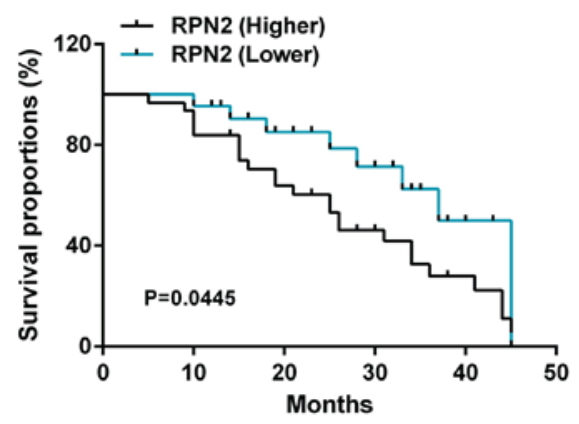

Figure 1. Expression of miR-128 and RPN2 in CRC tissues. (A) miR-128 expression was inhibited in CRC tissues. (B) RPN2 expression was significantly increased in CRC tissues. (C) Linear correlation of mRNA levels between miR-128 and RPN2 was assessed. (D) Survival analysis indicated that patients with CRC with increased levels of RPN2 survived for shorter periods of time compared with the patients with decreased RPN2 expression levels. Data are expressed as the mean \pm standard deviation from three independent experiments. ${ }^{* *} \mathrm{P}<0.01$ vs. normal tissues. miR, microRNA; RPN2, Ribophorin-II; $\mathrm{CRC}$, colorectal cancer.

bands were detected using enhanced chemiluminescence detection reagents (Amersham; GE Healthcare, Chicago, IL, USA). Band densities were quantified by densitometry Image $\mathrm{Lab}^{\mathrm{TM}}$ Software version 4.1 (Bio-Rad Laboratories, Inc.).

The antibodies used were as follows: Rabbit anti-RPN2 (Abcam, Cambridge, UK; cat. no., ab64467; 1:1,000 dilution); anti-p53 (Abcam; cat. no., ab131442; 1:1,000 dilution); anti-Cyclin D1 (Abcam; cat. no., ab226977; 1:2,000 dilution); anti-MMP-2 (Abcam; cat. no., ab37150; 1:1,000 dilution); anti-MMP-9 (Abcam; cat. no., ab73734; 1:1,000 dilution); anti-MTA1 (Abcam; cat. no., ab71153; 1:2,000 dilution); anti-E-cadherin (Abcam; cat. no., ab15148; 1:500 dilution); anti-TIMP2 (Abcam; cat. no., ab180630; 1:1,000 dilution); and anti-GAPDH (Abcam; cat. no., ab9485; 1:2,000 dilution).

Bioinformatics target prediction and luciferase reporter assays. Bioinformatics target prediction tools, including Target Scan (http://www.targetscan.org/), PicTar (http://pictar. mdc-berlin.de/) and miRNA targets (http://cbio.mskcc. $\mathrm{org} / \mathrm{mimaviewer} /$ ) were used to define the potential targets of miR-128 in 3'-UTR fragment of RPN2.

The miR-128-3p binding sequence of the RPN2 3'-UTR fragment was intentionally mutated using the Gene Tailor Site-Directed Mutagenesis System (Invitrogen; Thermo Fisher Scientific, Inc.), according to the protocol of the manufacturer. Then, the human RPN2 3'-UTR or mutated RPN2 3'-UTR sequences were ligated into the luciferase reporter vector pGL3-basic plasmid (Promega Corporation, Madison, WI, USA), to obtain theRPN2 3'-UTR and Mut-RPN2 3'-UTR recombinant luciferase reporter plasmids, respectively.
Then, $5 \times 10^{3} 293$ cells were seeded in 96-well plates and co-transfected with $0.2 \mu \mathrm{g}$ luciferase reporter plasmid (RPN2 3'-UTR, or Mut-RPN2 3'-UTR plasmid) and $50 \mathrm{nmol} / 1$ miRNAs [miR-128-3p or non-specific sequence (NC)], using Lipofectamine ${ }^{\circledR} 2000$. Following $24 \mathrm{~h}$ of incubation at $37^{\circ} \mathrm{C}$, cells were harvested by centrifugation $(5,000 \mathrm{x} \mathrm{g})$ at $4^{\circ} \mathrm{C}$ for $20 \mathrm{~min}$, and luciferase activities were measured using the Dual-Glo Luciferase Reporter Assay System (Promega Corporation) according to the protocol of the manufacturer. Data were normalized to the Renilla luciferase activity.

Statistical analysis. Statistical analyses were performed using SPSS 22.0 software (IBM Corp., Armonk, USA). Each experiment was repeated in triplicate, with all data presented as mean \pm standard deviation. A one-way analysis of variance followed by a Tukey's post-hoc test was used to compare either two or multiple groups. The $\chi^{2}$ test was used to compare categorical variables. A Spearman rank correlation coefficient was used to analyze the correlation between variables. Kaplan-Meier and log-rank test were used for survival analysis. $\mathrm{P}<0.05$ was considered to indicate a statistically significant difference. $\mathrm{P}<0.01$ was considered to indicate a particularly significant difference.

\section{Results}

miR-128 and RPN2 levels in CRC tissues. The present study included a total of 53 patients, with a median age of 57 years. The expression of miR-128 and RPN2 in CRC tissues and adjacent normal tissues was assessed using RT-qPCR. 
Compared with normal tissues, the levels of miR-128 in CRC tissues were significantly decreased, while the expression of RPN2 was increased in CRC tissues ( $\mathrm{P}<0.01$; Fig. 1A and B). The correlation analysis indicated a marked negative association between the expression of miR-128 and RPN2, which suggested that miR-128 was likely to be significant in the control of RPN2 regulation (Fig. 1C). A total of 70\% (37/53) patient tissues exhibited increased RPN2 expression in tumor tissues compared with adjacent normal tissues, were placed in the high RPN2 expression group. Patients with decreased RPN2 expression in tumor tissues, compared with adjacent normal tissues, were placed in the low RPN2 expression group. As demonstrated in Table II, compared with adjacent tissue, the increased RPN2 and corresponding reduced miR-128 were significantly associated with poorly-differentiated histology $(\mathrm{P}=0.042)$, advanced disease stages $(\mathrm{P}=0.004)$ and lymph node metastasis $(\mathrm{P}=0.013)$. It was also detected that the survival rate was decreased in patients with CRC with an increased expression of RPN2, and corresponding decreased miR-128 levels, compared with patients with decreased levels of RPN2 and corresponding increased miR-128 levels ( $\mathrm{P}=0.445$, Fig. 1D).

miR-128 and RPN2 levels in CRC cell lines. Decreased miR-128mRNA levels, as determined by RT-qPCR, along with increased RPN2 mRNA and protein levels, as determined by RT-qPCR and western blot analysis, were also evaluated in 4 human CRC HT29, SW480, SW620, and HCT116 cell lines in comparison with the normal CRC NCM460 cell line. The most significant variations among the CRC cell lines were detected in the HT29 cells, which was then selected to conduct subsequent experiments $(\mathrm{P}<0.05$; Fig. 2).

miR-128 downregulates $m R N A$ and protein expression levels of RPN2 in HT29CRC cells. The miR-128 levels in the miR-128 group, determined by RT-qPCR, were increased 2-fold in comparison with the control and mock groups $(\mathrm{P}<0.05$; Fig. 3A). The effect of miR-128 mimics on RPN2 levels was subsequently evaluated using RT-qPCR and western blot analysis (Fig. 3B and C). Aberrant increased miR-128 levels downregulated the mRNA and protein expression levels of RPN2. As indicated in Fig. 3C, the overexpression of miR-128 resulted in a $\sim 4 \%$ decrease in RPN2 protein expression compared with the mock and control groups $(\mathrm{P}<0.05)$.

miR-128 inhibits cell proliferation, migration and invasion of HT29CRC cells. Then, the effect of miR-128 on the biological processes of HT29 cells including cell proliferation, migration and invasion was detected (Fig. 3D-F). In the miR-128 group, cell proliferation was significantly inhibited by miR-128 overexpression compared with the mock and control groups (37\%; $\mathrm{P}<0.05$; Fig. 3D). Additionally, increased levels of miR-128 attenuated the cell migration and invasion rates; the inhibition rates were $\sim 31 \%$ and $33 \%$, respectively ( $\mathrm{P}<0.01$; Fig. $3 \mathrm{E}$ and $\mathrm{F}$ ). The results suggested that miR-128 serves important roles in CRC.

miR-128 inhibits the Akt-p53-cyclin signal pathway in HT29 cells. To investigate the effect of miR-128 and RPN2 on the Akt-p53-Cyclin pathway in CRC cells, the phosphorylation levels of Akt and mRNA and protein levels of p53 and cyclin D1 were assessed in miR-128, mock and control groups
Table II. Association between RPN2 and clinical data of patients with colorectal cancer.

\begin{tabular}{lcc}
\hline & $\begin{array}{c}\text { RPN2 expression } \\
\text { Factors }\end{array}$ & P-value \\
\hline Age, years & & 0.981 \\
$<50$ & $14 / 6$ & \\
$\geq 50$ & $23 / 10$ & 0.726 \\
Sex & & \\
Male & $26 / 12$ & \\
Female & $11 / 4$ & $0.042^{\mathrm{a}}$ \\
Histological grade & & \\
Well-moderate & $10 / 9$ & $0.004^{\mathrm{b}}$ \\
Poor & $27 / 7$ & \\
TNM & & \\
I-II & $10 / 11$ & \\
III-IV & $27 / 5$ & \\
M stage & & \\
M0 & $8 / 9$ & \\
M1 & $29 / 7$ & \\
\hline
\end{tabular}

${ }^{\mathrm{a}} \mathrm{P}<0.05$ and ${ }^{\mathrm{b}} \mathrm{P}<0.01$ by $\chi^{2}$ test; TNM, Tumor-Node-Metastasis; RPN2, Ribophorin-II.

(Fig. 4). The overexpression of miR-128, combined with low levels of RPN2, inhibited Akt phosphorylation, downregulated cyclin D1 and upregulated p53 expression at mRNA and protein levels $(\mathrm{P}<0.05)$.

miR-128 affects the expression of invasion-associated factors including MMP-2, MMP-9, MTA1, E-cadherin and TIMP 2 in HT29 cells. The expression levels of MMP-2, MMP-9, MTA1, E-cadherin and TIMP2 was affected by miR-128 overexpression and RPN2 downregulation (Fig. 5). miR-128 mimic transfection inhibited the expression of MMP-2, MMP-9 and MTA1, and promoted the expression of E-cadherin and TIMP2 at mRNA and protein levels $(\mathrm{P}<0.05)$.

Bioinformatics target prediction and luciferase reporter assays. The target prediction databases indicated that one highly conserved miR-128 binding site was present in the RPN2 3'-UTR (Fig. 6A and B). Direct interaction between miR-128 and RPN2 was verified by dual-luciferase activity assay. miR-128 mimic transfection significantly inhibited the relative luciferase activity of RPN2 3'-UTR in 293 cells $(\mathrm{P}<0.05)$, while there was no significant difference among the control, NC and miR-128 + Mut-RPN2 3'-UTR groups ( $\mathrm{P}>0.05$; Fig. 6C).

\section{Discussion}

Colorectal cancer (CRC) is a malignancy with high mortality rates worldwide, which may be due to high metastasis rates $(33,34)$. Previous studies have suggested that the aberrant expression of miRNAs, including miR-128, was identified to 
A

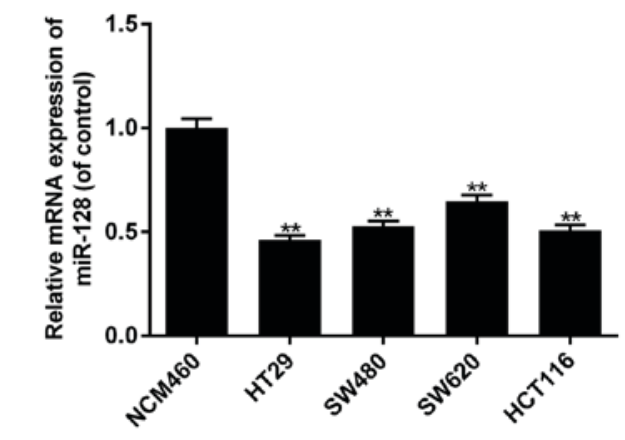

C

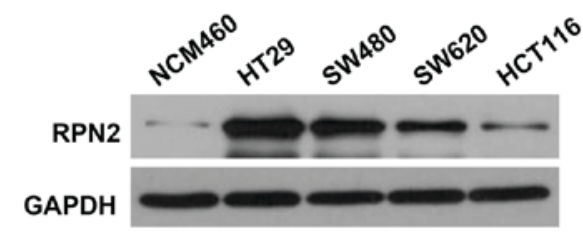

B

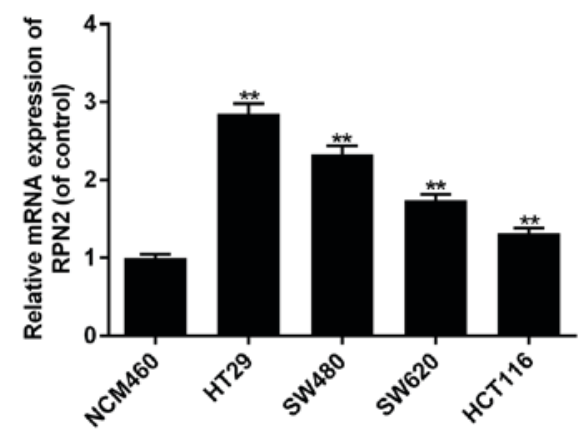

D

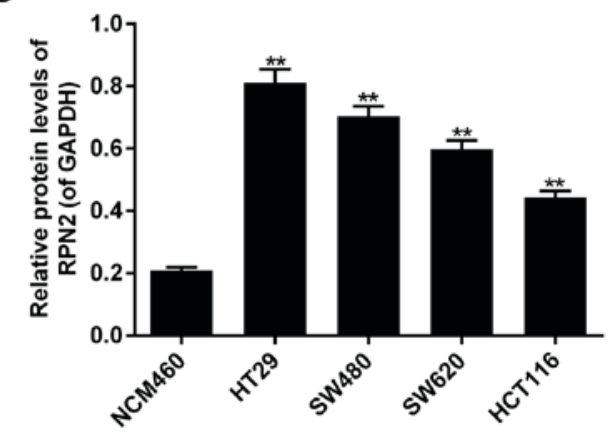

Figure 2. Expression of miR-128 and RPN2 in CRCHT29, SW480, SW620 and HCT116 cell lines was assessed. (A) The miR-128 levels in CRC cell lines were significantly decreased, compared with the normal NCM460 cell line. (B) The mRNA levels of RPN2 in CRC cell lines were significantly increased, compared with normal NCM460 cells. (C) Western blot analysis of RPN2 protein expression in CRC and normal cell lines. (D) The protein levels of RPN2 in CRC cell lines were significantly increased compared with normal NCM460 cells. Data are expressed as the mean \pm standard deviation from three independent experiments. ${ }^{* *} \mathrm{P}<0.01$ vs. normal cells. RPN2, Ribophorin-II; CRC, colorectal cancer.

be associated with clinical CRC development and metastasis by regulating target genes and mediating protein expression in cancer-associated pathways (35-37). Concomitantly, RPN2 has been suggested to be closely associated with the tumorigenesis of CRC $(38,39)$. However, whether miR-128 exhibits a direct regulatory effect on RPN2 in CRC cell proliferation and metastasis remains unknown.

In the present study, it was identified that the aberrant expression of miR-128 was negatively associated with RPN2 in CRC tissues and cell lines including HT29, SW480, SW620, and HCT116 cells. The increased RPN2 levels and corresponding decreased miR-128 levels were significantly associated with poorly-differentiated histology, advanced disease stages and lymph node metastasis in patients with CRC. In addition, the survival rate of patients with CRC was associated with RPN2 levels. These data suggest that miR-128 and RPN2 serve critical roles in CRC development and tumor metastasis.

In order to detect the molecular mechanisms of miR-128 and RPN2 regulation during CRC development, the miR-128 overexpression model was constructed by miR-128 mimic transfection in HT29 cells, in order to induce the highest RPN2 expression and lowest miR-128 expression levels. The results of this assay verified that miR-128 overexpression downregulated the mRNA and protein expression levels of RPN2, and significantly inhibited CRC cell proliferative, migratory and invasive abilities. It suggested that the negative regulatory role of miR-128 on RPN2 may serve as a suppressor of CRC development and metastasis.
As an essential subunit of the oligosaccharide transferase complex, RPN2 induces cell signal recognition and transduction and cooperates with numerous signal pathways, including the phosphatidylinositol 3-kinase/Akt signaling pathway, which is suggested to be closely associated with tumorigenesis $(40,41)$. The phosphorylation of Akt will activate downstream target genes to regulate the cell cycle, proliferation and migration (42). This is achieved primarily through activation of cyclin D1 by phosphorylated Akt to promote cell cycle G1/S transition (43). Cyclin D1, a downstream target of the Akt-p53 signaling pathway, is a key in nuclear transcription factor in cell cycle regulation. The activation of Akt and Cyclin D1 has been demonstrated to be associated with CRC occurrence and development (44). The present study identified that aberrant high miR-128 expression, along with low RPN2 expression, decreased the phosphorylation level of Akt, downregulated the mRNA and protein levels of cyclin D1 and promoted p53 expression levels markedly, which indicated an inhibition of theAkt-p53-cyclin signal pathway by miR-128 mimics. It suggested that the suppressive function of miR-128 mimics on the migratory ability of HT29 cells may depend on the inhibition of Akt-p53-cyclin signal pathway.

To additionally verify this hypothesis, the effect of the ectopic expression of miR-128 on epithelial-mesenchymal transition (EMT) and metastasis-associated genes including E-cadherin, MMP-2, MMP-9, MTA1 andTIMP2 were evaluated. EMT is an essential process for cell metastasis (45), in which cancer cells obtain migratory capabilities to enter 
A

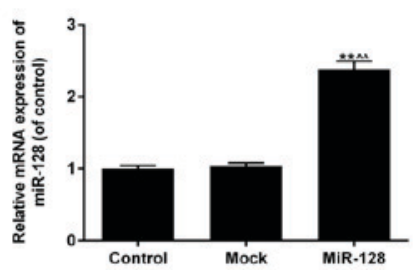

C

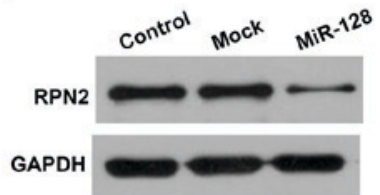

E

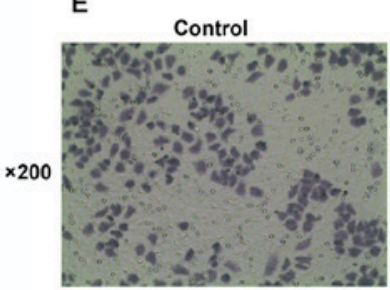

$\mathbf{F}$

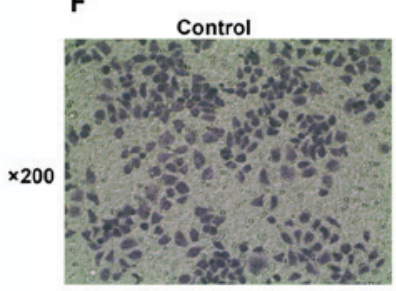

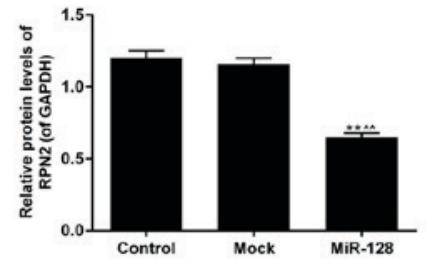

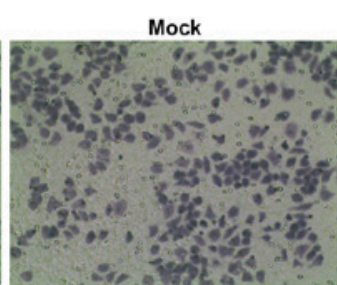

Mock

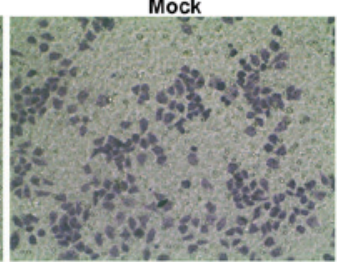

B

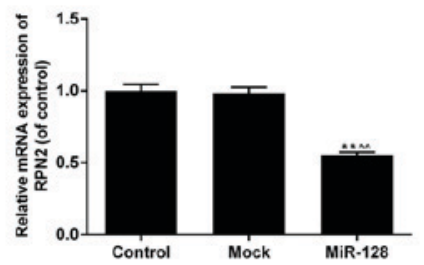

D
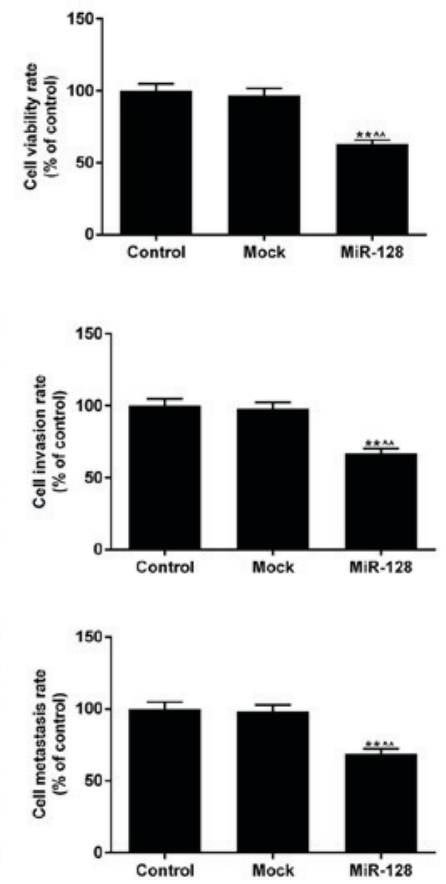

Figure 3. miR-128 overexpression affects cell proliferation, migration and invasion. (A) Ectopic miR-128 expression was successfully established in a transfection model. (B) Ectopic miR-128 expression decreased the RPN2 mRNA level. (C) The expression of RPN2 protein was inhibited by miR-128 mimics. (D) miR-128 overexpression decreased the proliferative ability of HT29 cells. (E) miR-128 attenuated the cell migration rate in HT29 cells (magnification, $\mathrm{x} 200$ ). (F) miR-128 decreased the cell invasion rate in HT29 cells (magnification, $\mathrm{x} 200$ ). Data are expressed as the mean \pm standard deviation from three independent experiments. ${ }^{* *} \mathrm{P}<0.01$ vs. control and ${ }^{\wedge} \mathrm{P}<0.01$ vs. mock cells. miR, microRNA; RPN2, Ribophorin-II.

A

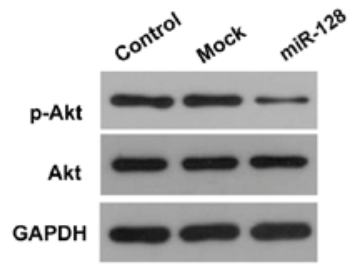

C

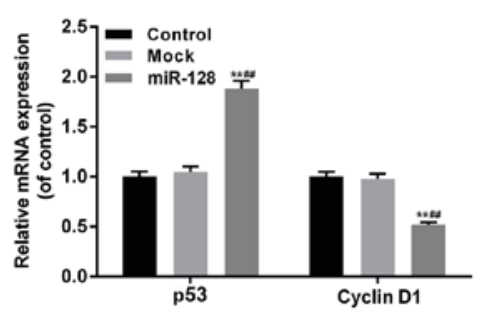

B

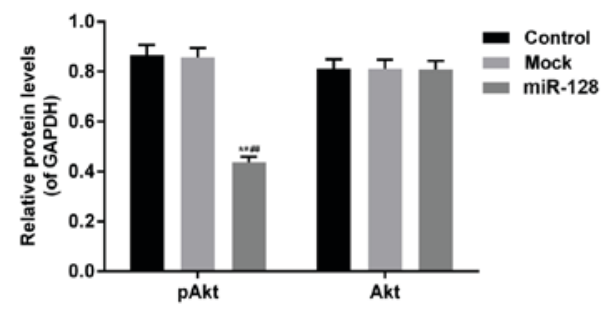

E

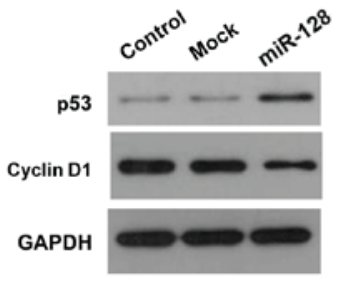

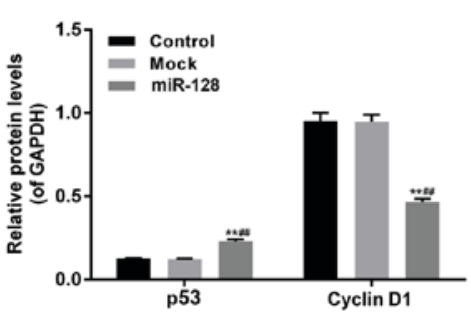

Figure 4. miR-128 overexpression inhibits the Akt-p53-cyclin pathway. (A) Ectopic miR-128 expression was successfully established in a transfection model. (B) Ectopic miR-128 expression inhibited the phosphorylation levels of Akt. (C) The mRNA levels of cyclin D1 were decreased, while levels of p53 were increased in miR-128 overexpressed cells. (D) Western blot analysis of the p53 and cyclin D1 protein levels. (E) The protein levels of cyclin D1 were decreased, while the levels of p53 were increased in miR-128 overexpressing cells. Data are expressed as the mean \pm standard deviation from three independent experiments. ${ }^{* *} \mathrm{P}<0.01$ vs. control and ${ }^{\wedge} \mathrm{P}<0.01$ vs. mock. miR, microRNA; Akt, protein kinase $\mathrm{B}$; p53, tumor protein 53. 
A

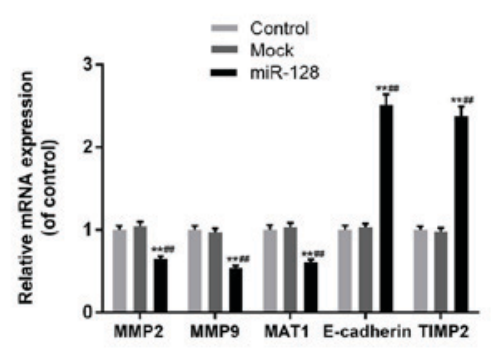

B

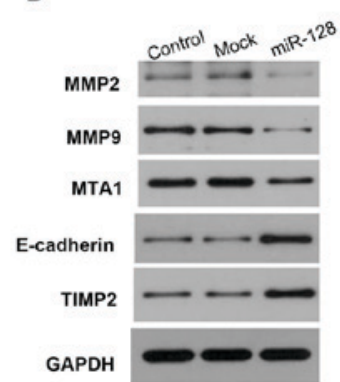

c

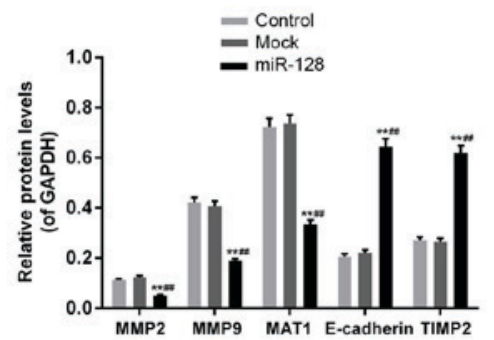

Figure 5. miR-128 overexpression affects the expression of cell invasion-associated factors. (A) Aberrant increased miR-128 levels and inhibited Ribophorin-II expression downregulated the mRNA expression of MMP-2, MMP-9 and MTA1, and upregulated E-cadherin and TIMP2 mRNA levels. (B and C) MMP-2, MMP-9 and MTA1 proteins were downregulated, while E-cadherin and TIMP2 proteins were increased in the miR-128 mimic-transfected cells. Data are expressed as the mean \pm standard deviation from three independent experiments. ${ }^{* *} \mathrm{P}<0.01$ vs. control, ${ }^{\wedge} \mathrm{P}<0.01$ vs. mock. miR, microRNA; MMP, matrix metalloproteinase; MTA1, metastasis-associated protein 1; E-cadherin, epithelial cadherin; TIMP1, Tissue inhibitor of metalloproteinases 2.

A

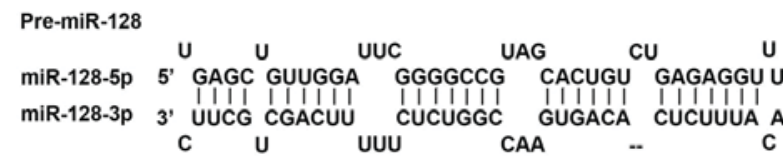

B

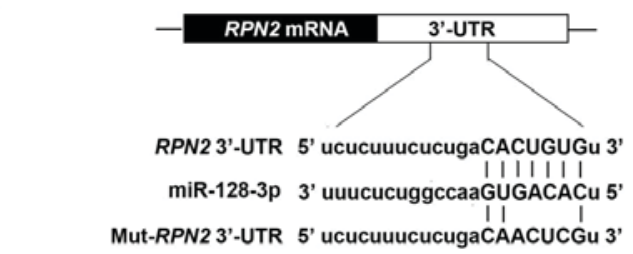

C

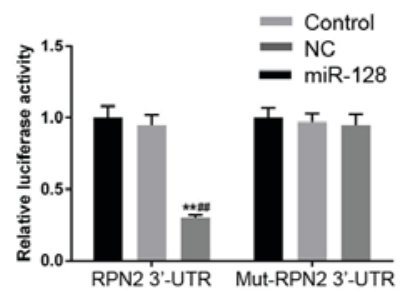

Figure 6. Binding site prediction and luciferase report assay. (A) The cervical-loop structures of miR-128. (B) Putative targets predicted by Target Scan. (C) Relative luciferase activity resulted from binding of RPN2 3'-UTR reporter and miR-128. ${ }^{* *} \mathrm{P}<0.01$ vs. control, ${ }^{\wedge} \mathrm{P}<0.01$ vs. NC. miR, microRNA; RPN2, Ribophorin-II; UTR, untranslated region; NC, negative control; Mut, mutant.

into circulatory system via the extracellular matrix and basement membrane of blood vessels (46). E-cadherin is the marker of epithelial cells. As a cell adhesion molecule, the protein complexes of E-cadherin combine with actin cytoskeletons to weaken cell-cell adhesion and induce migration and invasion of tumor cells (47). MMPs are structurally analogous, zinc-dependent endopeptidases (48). MMPs and their inhibitors (TIMPs) serve important roles in extracellular matrix degradation $(49,50)$, which may induce tumor invasion and metastasis (51). MMP2 and MMP9 are able to degrade the primary component of type IV collagen to induce basement membranes degradation (52), which results in cell migration and eventual tumor metastasis. MMPs serve important roles in EMT-associated regulation of cell migration (53). The mRNA expression of MMP9 was verified to be notably improved during murine colitis-associated cancer progression following administering azoxymethane and dextran sulfate sodium ingestion (54). MTA1 was demonstrated to be persistently highly expressed in lymph nodes metastasis in non-small cell lung and breast cancer, CRC and pancreatic cancer. It may inhibit the expression of tumor suppressors and contribute to cell migration and invasion (55). In the present study, it was confirmed that the expression levels of MMP-2, MMP-9, MTA1, E-cadherin and TIMP2 were significantly affected by miR-128 overexpression not only at mRNA levels but also at protein levels. Cells transfected with miR-128 mimics expressed decreased levels of MMP-2, MMP-9 and MTA1, but increased levels of E-cadherin and TIMP2, which inhibited cell migration. It was also revealed that the regulation of metastasis-associated proteins was necessary in the molecular mechanisms underlying the miR-128-associated modification of HT29 cell metastasis.

To comprehensively investigate the association between miR-128 and RPN2 in regulating CRC cell proliferation and migration, additional experiments were performed. According to the bioinformatics analysis, it was identified that the RPN2 3'-UTR contained the binding site of miR-128. Luciferase reporter gene analysis confirmed that RPN2 was a direct target of miR-128: The RPN2 3'-UTR or Mut-RPN2 3'-UTR recombinant plasmids were co-transfected with miR-128 mimics into 293 cells. The results demonstrated that the miR-128 mimics significantly inhibited the relative luciferase activity of RPN2 3'-UTR cells, but exhibited no effect on the Mut-RPN2 3'-UTR plasmid. This confirmed that miR-128 directly targeted the 3'-UTR of RPN2. It also suggested that miR-128 targeted RPN2 during the regulatory process of CRC cell proliferation and migration.

In conclusion, the results of the present study suggested that miR-128 was a specific negative regulator of RPN2, which attenuated colorectal cancer cell proliferation and migration. This regulation may be associated with the Akt-p53 signal pathway and metastasis-associated factors. These data may provide novel evidence for potential target biomarkers in the treatment of colorectal cancer. 


\section{Acknowledgements}

Not applicable.

\section{Funding}

The present study was supported by Guangdong Provincial Science and Technology Plan (grant no. 2017A020215036).

\section{Availability of data and materials}

All data generated or analyzed during this study are included in this published article.

\section{Authors' contributions}

TZ constructed miR-128 overexpressed cells and detected the invasion and migration abilities. LW studied the expression levels of RPN2 in CRC cells. QW investigated the effect on P53/Cyclin D1. ZJ, NM and YL collected the tissue samples and conducted associated RT-qPCR and Western blot analysis. WC, ZH, WG investigated the levels of metastasis associated factors. SC conceived the study and drafted the manuscript.

\section{Ethics approval and consent to participate}

All tissue samples from patients were collected and protocols were performed according to the procedures approved by the Institutional Review Board of the Independent Ethics Committee of the Sixth Affiliated Hospital of Sun Yat-sen University (approval no. BZ20153587). All patients provided informed consent.

\section{Patient consent for publication}

Not applicable.

\section{Competing interests}

The authors declare that they have no competing interests.

\section{References}

1. Kamangar F, Dores GM and Anderson WF: Patterns of cancer incidence, mortality, and prevalence across five continents: Defining priorities to reduce cancer disparities in different geographic regions of the world. J Clin Oncol 24: 2137-2150, 2006.

2. Shroff J, Thosani N, Batra S, Singh H and Guha S: Reduced incidence and mortality from colorectal cancer with flexible-sigmoidoscopy screening: A meta-analysis. World J Gastroenterol 20: 18466-18476, 2014.

3. Chen W, Zheng R, Zeng H and Zhang S: The updated incidences and mortalities of major cancers in China, 2011. Chin J Cancer 34: 502-507, 2015.

4. Labianca R and Merelli B: Screening and diagnosis for colorectal cancer: Present and future. Tumori 96: 889-901, 2010.

5. Levi F, Lucchini F, Negri E, Zatonski W, Boyle P and La Vecchia C: Trends in cancer mortality in the European Union and accession countries, 1980-2000. Ann Oncol 15: 1425-1431, 2004.

6. Chouhan V, Mansoor E, Parasa S and Cooper GS: Rates of prevalent colorectal cancer occurrence in persons 75 years of age and older: A population-based national study. Dig Dis Sci 63: 1929-1936, 2018.
7. Kumar A, Cherukumilli M, Mahmoudpour SH, Brand K and Bandapalli OR: ShRNA-mediated knock-down of CXCL8 inhibits tumor growth in colorectal liver metastasis. Biochem Biophys Res Commun 500: 731-737, 2018.

8. Peng Y, Zhao BC, Kang Q, Liu J, Chen C, Li BS, Xie YP and Wu Q: Colorectal cancer preventive effect of combined administration of phenolic acids and supercritical extracts from Angelica sinensis. Zhongguo Zhong Yao Za Zhi 43: 1235-1240, 2018 (In Chinese).

9. Costa FF, Bischof JM, Vanin EF, Lulla RR, Wang M, Sredni ST, Rajaram V, Bonaldo Mde F, Wang D, Goldman S, et al: Identification of microRNAs as potential prognostic markers in ependymoma. PLoS One 6: e25114, 2011.

10. Gattolliat CH, Thomas L, Ciafrè SA, Meurice G, Le Teuff G, Job B, Richon C, Combaret V, Dessen P, Valteau-Couanet D, et al: Expression of miR-487b and miR-410 encoded by $14 \mathrm{q} 32.31$ locus is a prognostic marker in neuroblastoma. Br J Cancer 105: 1352-1361, 2011.

11. Haller F, von Heydebreck A, Zhang JD, Gunawan B, Langer C, Ramadori G, Wiemann S and Sahin O: Localization- and mutation-dependent microRNA (miRNA) expression signatures in gastrointestinal stromal tumours (GISTs), with a cluster of co-expressed miRNAs located at 14q32.31. J Pathol 220: 71-86, 2010.

12. Lavon I, Zrihan D, Granit A, Einstein O, Fainstein N, Cohen MA, Cohen MA, Zelikovitch B, Shoshan Y, Spektor S, et al: Gliomas display a microRNA expression profile reminiscent of neural precursor cells. Neuro Oncol 12: 422-433, 2010.

13. Vasudevan S, Tong Y and Steitz JA: Switching from repression to activation: microRNAs can up-regulate translation. Science 318 : 1931-1934, 2007.

14. Gong J, Zhang JP, Li B, Zeng C, You K, Chen MX, Yuan Y and Zhuang SM: MicroRNA-125b promotes apoptosis by regulating the expression of Mcl-1, Bcl-w and IL-6R. Oncogene 32: 3071-3079, 2013

15. Corté H, Manceau G, Blons H and Laurent-Puig P: MicroRNA and colorectal cancer. Dig Liver Dis 44: 195-200, 2012.

16. Wu N, Zhao X, Liu M, Liu H, Yao W, Zhang Y, Cao S and Lin X: Role of microRNA-26b in glioma development and its mediated regulation on EphA2. PLoS One 6: e16264, 2011.

17. Ai F, Zhang X, Li X, Qin Z, Ye Q, Tian L, Tang A, Li N, Li G, Ma J and Shen S: Up-regulation of matrix metalloproteinases in a mouse model of chemically induced colitis-associated cancer: The role of microRNAs. Oncotarget 6: 5412-5425, 2015.

18. Takahashi Y, Iwaya T, Sawada G, Kurashige J, Matsumura T, Uchi R, Ueo H, Takano Y, Eguchi H and Sudo T: Up-regulation of NEK2 by MicroRNA-128 methylation is associated with poor prognosis in colorectal cancer. Ann Surg Oncol 21: 205-212, 2014.

19. Cao Y, Pang $\mathrm{H}$ and Chen C: MicroRNA-128 inhibits invasion of colon cancer cells. Zhejiang Med J, 2016 (In Chinese).

20. Ciafrè SA, Galardi S, Mangiola A, Ferracin M, Liu CG, Sabatino G, Negrini M, Maira G, Croce CM and Farace MG: Extensive modulation of a set of microRNAs in primary glioblastoma. Biochem Biophys Res Commun 334: 1351-1358, 2005.

21. Tutar Y: miRNA and cancer; computational and experimental approaches. Curr Pharm Biotechnol 15: 429, 2014.

22. Godlewski J, Nowicki MO, Bronisz A, Williams S, Otsuki A, Nuovo G, Raychaudhury A, Newton HB, Chiocca EA and Lawler S: Targeting of the Bmi-1 oncogene/stem cell renewal factor by microRNA-128 inhibits glioma proliferation and self-renewal. Cancer Res 68: 9125-9130, 2008.

23. Koulich E, Li X and DeMartino GN: Relative structural and functional roles of multiple deubiquitylating proteins associated with mammalian 26S proteasome. Mol Biol Cell 19: 1072-1082, 2008.

24. Fujita Y, Yagishita S, Takeshita F, Yamamoto Y, Kuwano K and Ochiya T: Prognostic and therapeutic impact of RPN2-mediated tumor malignancy in non-small-cell lung cancer. Oncotarget 6: $3335-3345,2015$.

25. Honma K, Iwao-Koizumi K, Takeshita F, Yamamoto Y, Yoshida T, Nishio K, Nagahara S, Kato K and Ochiya T: RPN2 gene confers docetaxel resistance in breast cancer. Nat Med 14: 939-948, 2008.

26. Fujita Y, Takeshita F, Mizutani T, Ohgi T, Kuwano K and Ochiya T: A novel platform to enable inhaled naked RNAi medicine for lung cancer. Sci Rep 3: 3325, 2013.

27. Takahashi RU, Takeshita F, Honma K, Ono M, Kato K and Ochiya T: Ribophorin II regulates breast tumor initiation and metastasis through the functional suppression of GSK3 $\beta$. Sci Rep 3: 2474, 2013. 
28. Zhao Y, Deng Y, Peng J, Sui Q, Lin J, Qiu M and Pan Z: Does the preoperative prognostic nutritional index predict survival in patients with liver metastases from colorectal cancer who underwent curative resection? J Cancer 9: 2167-2174, 2018.

29. Hendricks A, Eggebrecht GL, Bernsmeier A, Geisen R, Dall K, Trauzold A, Becker T, Kalthoff H, Schafmayer C, Röder C and Hinz S: Identifying patients with an unfavorable prognosis in early stages of colorectal carcinoma. Oncotarget 9: 27423-27434, 2018.

30. Han HQ, Liu T, Zhao LZ, Qi F and Wang PZ: Clinical value of the sixth edition TNM stages analysing prognosis of colorectal cancer. Zhonghua Yi Xue Za Zhi 86: 819-821, 2006 (In Chinese).

31. Chen JX, Tang XD, Xiang DB, Dong XL, Peng FY and Sun GY: TNM stages and prognostic features of colorectal mucinous adenocarcinomas: A meta analysis. Asian Pac J Cancer Prev 13: 3427-3430, 2012.

32. Livak KJ and Schmittgen TD: Analysis of relative gene expression data using real-time quantitative PCR and the 2(-Delta Delta C(T)) method. Methods 25: 402-408, 2001

33. D'Haene N, Fontanges Q, De Nève N, Blanchard O, Melendez B, Delos M, Dehou MF, Maris C, Nagy N, Rousseau E, et al: Clinical application of targeted next-generation sequencing for colorectal cancer patients: A multicentric Belgian experience. Oncotarget 9: 20761-20768, 2018.

34. Sun J, Hu J, Wang G, Yang Z, Zhao C, Zhang X and Wang J: LncRNA TUG1 promoted KIAA1199 expression via miR-600 to accelerate cell metastasis and epithelial-mesenchymal transition in colorectal cancer. J Exp Clin Cancer Res 37: 106, 2018.

35. Feng H, Xu M, Zhang Y, Han B, Wang J and Sun P: Identification of differentially expressed MicroRNAs involved in the pathogenesis of colorectal cancer. Clin Lab 64: 797-804, 2018.

36. Li J, Xu J, Yan X, Jin K, Li W and Zhang R: MicroRNA-485 plays tumour-suppressive roles in colorectal cancer by directly targeting GAB2. Oncol Rep 40: 554-564, 2018

37. Sun W, Wang X, Li J, You C, Lu P, Feng H, Kong Y, Zhang H, Liu Y, Jiao R, et al: MicroRNA-181a promotes angiogenesis in colorectal cancer by targeting SRCIN1 to promote the SRC/VEGF signaling pathway. Cell Death Dis 9: 438, 2018.

38. Zhang J, Yan B, Späth SS, Qun H, Cornelius S, Guan D, Shao J, Hagiwara K, Van Waes C, Chen Z, et al: Integrated transcriptional profiling and genomic analyses reveal RPN2 and HMGB1 as promising biomarkers in colorectal cancer. Cell Biosci 5: 53 , 2015.

39. Li H, Al-Japairai K, Tao Y and Xiang Z: RPN2 promotes colorectal cancer cell proliferation through modulating the glycosylation status of EGFR. Oncotarget 8: 72633-72651, 2017.

40. Liu GL, Yang HJ, Liu B and Liu T: Effects of microrna-19b on the proliferation, apoptosis, and migration of Wilms' Tumor cells via the PTEN/PI3K/AKT signaling pathway. J Cell Biochem 118: 3424-3434, 2017

41. Lu X, Lv S, Mi Y, Wang L and Wang G: Neuroprotective effect of miR-665 against sevoflurane anesthesia-induced cognitive dysfunction in rats through PI3K/Akt signaling pathway by targeting insulin-like growth factor 2 . Am J Transl Res 9: 1344-1356, 2017

42. Manning BD and Cantley LC: AKT/PKB signaling: Navigating downstream. Cell 129: 1261-1274, 2007.

43. Liang J and Slingerland JM: Multiple roles of the PI3K/PKB (Akt) pathway in cell cycle progression. Cell Cycle 2: 339-345, 2003.
44. Liu Y, Bi T, Wang Z, Wu G, Qian L, Gao Q and Shen G: Oxymatrine synergistically enhances antitumor activity of oxaliplatin in colon carcinoma through PI3K/AKT/mTOR pathway. Apoptosis 21: 1398-1407, 2016.

45. Sabe H: Cancer early dissemination: Cancerous epithelial-mesenchymal transdifferentiation and transforming growth factor beta signalling. J Biochem 149: 633-639, 2011.

46. Acloque H, Adams MS, Fishwick K, Bronner-Fraser M and Nieto MA: Epithelial-mesenchymal transitions: The importance of changing cell state in development and disease. J Clin Invest 119: 1438-1449, 2009.

47. Gao H, Lan X, Li S and Xue Y: Relationships of MMP-9, E-cadherin, and VEGF expression with clinicopathological features and response to chemosensitivity in gastric cancer. Tumour Biol 39: 1010428317698368, 2017.

48. Visse R and Nagase H: Matrix metalloproteinases and tissue inhibitors of metalloproteinases: Structure, function, and biochemistry. Circ Res 92: 827-839, 2003.

49. Knapinska AM, Estrada CA and Fields GB: The roles of matrix metalloproteinases in pancreatic cancer. Prog Mol Biol Transl Sci 148: 339-354, 2017.

50. Parrish AR: Matrix metalloproteinases in kidney disease: Role in pathogenesis and potential as a therapeutic target. Prog Mol Biol Transl Sci 148: 31-65, 2017.

51. Curran S and Murray GI: Matrix metalloproteinases: Molecular aspects of their roles in tumour invasion and metastasis. Eur J Cancer 36: 1621-1630, 2000.

52. Hendrix AY and Kheradmand F: The role of matrix metalloproteinases in development, repair, and destruction of the lungs. Prog Mol Biol Transl Sci 148: 1-29, 2017.

53. Tang G, Du R, Tang Z and Kuang Y: MiRNALet-7a mediates prostate cancer PC-3 cell invasion, migration by inducing epithelial-mesenchymal transition through CCR7/MAPK pathway. J Cell Biochem 119: 3725-3731, 2018.

54. Shang K, Bai YP, Wang C, Wang Z, Gu HY, Du X, Zhou XY Zheng CL, Chi YY, Mukaida N and Li YY: Crucial involvement of tumor-associated neutrophils in the regulation of chronic colitis-associated carcinogenesis in mice. PLoS One 7: e51848, 2012.

55. Tuncay Cagatay S, Cimen I, Savas B and Banerjee S: MTA-1 expression is associated with metastasis and epithelial to mesenchymal transition in colorectal cancer cells. Tumor Biol 34: 1189-1204, 2013

c) (i) $\odot$ This work is licensed under a Creative Commons cc ${ }_{\text {EY NO ND }}$ Attribution-NonCommercial-NoDerivatives 4.0 International (CC BY-NC-ND 4.0) License. 